\title{
Induction of immune tolerance in kidney transplant recipients
}

A letter published in the New England Journal of Medicine gives hope that a method of inducing immune tolerance might enable the discontinuation of antirejection medicines in kidney transplant recipients.

The chronic use of immunosuppressive medications required to prevent graft rejection in kidney transplant recipients is associated with many adverse effects, including increased risks of infection, diabetes and cancer. As pilot clinical studies have indicated that inducing immune tolerance in kidney transplant recipients might abolish the lifelong need for immunosuppression, Scandling and colleagues conducted a proof-of-concept study in 12 recipients of HLA-matched renal transplants.

The 12 renal transplant recipients received a conditioning regimen of 10 doses of total lymphoid irradiation targeted to their thymus, spleen and lymph nodes, along with five doses of rabbit antithymocyte globulin during the first 10 days after transplantation. On the $11^{\text {th }}$ day after transplantation the patients were given an intravenous injection of donor cells consisting of highly enriched CD $34^{+}$hematopoietic progenitor cells mixed with $\mathrm{CD}^{+} \mathrm{T}$ cells. The patients received mycophenolate mofetil for 1 month and ciclosporin for at least 6 months; ciclosporin was discontinued 6-17 months after transplantation if chimerism had persisted for $\geq 6$ months and there was no evidence of rejection or graft-versus-host disease.

The researchers found that antirejection drugs could be discontinued in eight patients (67\%). These patients were followed up for 12-36 months with no evidence of chronic rejection or graft injury on the basis of proteinuria, creatinine clearance or the histopathological scoring of repeat surveillance biopsy samples. One patient who had discontinued antirejection drugs for 36 months died suddenly while exercising, 4 months after a myocardial infarction. Four patients had to continue on immunosuppressive treatment owing to recurrence of focal segmental glomerulosclerosis $(n=1)$ or rejection episodes during ciclosporin tapering $(n=3)$.

"In conclusion, the majority of patients were able to discontinue antirejection medications, and all patients had excellent graft function at the last observation point," state the authors. "We are applying the protocol to patients who were mismatched for one HLA haplotype on the basis of the safety profile of the current study."

\section{Rebecca Ireland}

Original article Scandling, J. D., Busque, S., Shizuri, J. A. Engleman, E. G. \& Strober, S. Induced immune tolerance for kidney transplantation. N. Engl. J. Med. 365, 1359-1360 (2011)

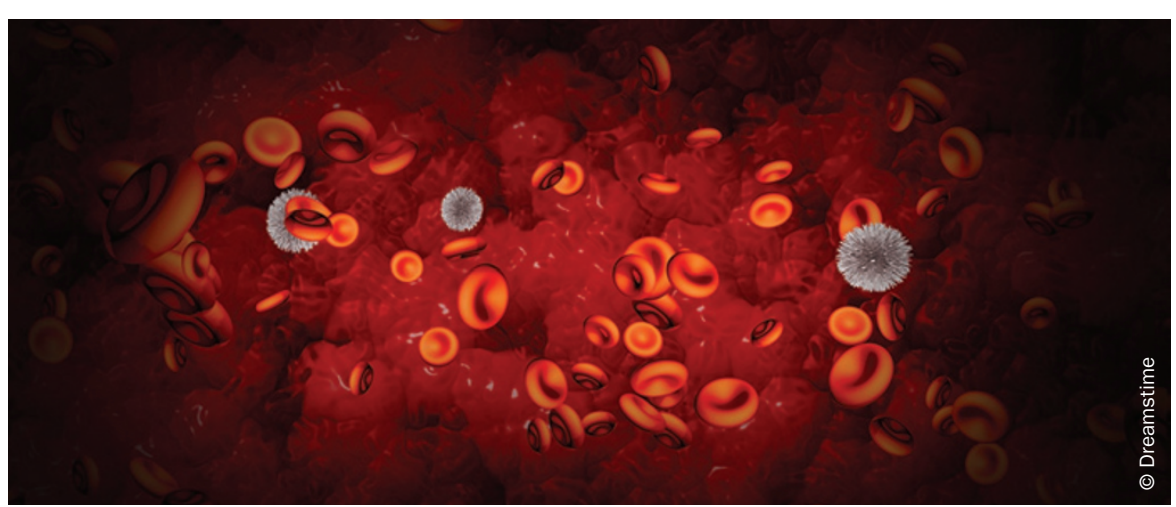

